\title{
Nitrofurantoin Susceptibility Pattern in Gram-Negative Urinary Isolates: In Need of Increased Vigilance
}

\author{
Garima Gautam ${ }^{1}$ Sanjib Gogoi ${ }^{1}$ Sonal Saxena ${ }^{2}$ Ravinder Kaur ${ }^{1}$ Megh Singh Dhakad ${ }^{1}$ \\ ${ }^{1}$ Department of Microbiology, Lady Hardinge Medical College and \\ Associated Hospitals, New Delhi, India \\ 2Department of Microbiology, Maulana Azad Medical College and \\ Associated Hospitals, New Delhi, India \\ Address for correspondence Ravinder Kaur, MD, Department \\ of Microbiology, Lady Hardinge Medical College and Associated \\ Hospitals, Lal Building 1st Floor, New Delhi 110001, India \\ (e-mail: drkaurarticles01@gmail.com).
}

J Lab Physicians 2021;13:252-256.

\begin{abstract}
Keywords

- nitrofurantoin

- disk diffusion

- antimicrobial susceptibility

- gram-negative bacteria

Nitrofurantoin is the first-line drug in the treatment of uncomplicated urinary tract infections (UTIs) and its use has increased exponentially in recent years.

Objectives This study aims to determine the susceptibility pattern of nitrofurantoin in gram-negative urinary isolates and to evaluate their bacteriological and epidemiological profile along with co-existing resistance to other important urinary antimicrobials.

Material and Methods This was a retrospective study conducted in a tertiary care hospital in New Delhi in which 500 gram-negative bacterial urinary isolates were evaluated. Records of antimicrobial susceptibility were reviewed from July to September 2019. Antimicrobial susceptibility was performed using the Kirby-Bauer disk diffusion method on Mueller Hinton agar and interpreted using CLSI 2019. Test for extended spectrum $\beta$-lactamase (ESBL) producers was done using double disk approximation test.

Statistical Analysis Data analysis was performed using the SPSS windows version 25.0 software.

Results Out of total 500 isolates, $20.17 \%(94)$ isolates were resistant (R) to nitrofurantoin and $9.01 \%$ (42) were found to be intermediate (I). Highest resistance was seen in Klebsiella sp. (44.61\%) and Escherichia coli (8.12\%). About $28.82 \%$ of the I/R isolates were of the pediatrics age group and most of the isolates belonged to females (64.69\%). High resistance was also seen against ampicillin (92.30\%), cefazolin (88.46\%), ceftazidime (73.0\%), and fluoroquinolones (65.38\%). Carbapenemase co-resistance was seen in $57.15 \%$ isolates whereas ESBL production was seen in $30.76 \%$ of $E$. coli and $12.06 \%$ of Klebsiella sp.

Conclusion Increase in multidrug resistance uropathogens along with a near absence of novel oral antibiotics has led to increased consumption of nitrofurantoin since its resistance has increased.
\end{abstract}

published online July 6, 2021
DOI https://doi.org/

$10.1055 / \mathrm{s}-0041-1731106$ ISSN $0974-2727$
(C)2021. The Indian Association of Laboratory Physicians.

This is an open access article published by Thieme under the terms of the Creative Commons Attribution-NonDerivative-NonCommercial-License, permitting copying and reproduction so long as the original work is given appropriate credit. Contents may not be used for commercial purposes, or adapted, remixed, transformed or built upon. (https://creativecommons.org/licenses/by-nc-nd/4.0/).

Thieme Medical and Scientific Publishers Pvt. Ltd. A-12, 2nd Floor, Sector 2, Noida-201301 UP, India 


\section{Key Message}

Nitrofurantoin susceptibility rate reflects the need for strict vigilance regarding nitrofurantoin use and regular monitoring of its susceptibility pattern.

\section{Introduction}

Increase in multidrug-resistant organisms has become an alarming situation around the globe. Resistance to last resort antibiotics such as carbapenems has also been increasing. ${ }^{1}$ This rise in resistance has been difficult to tackle due to lack of prudent antimicrobial use and susceptibility surveillance in many areas and lack of development of newer antibiotics.

Following the overuse of trimethoprim-sulfamethoxazole and fluroquinolones, most uropathogens are now resistant to these oral drugs. ${ }^{2}$ Nitrofurantoin (NFT) has been used for more than 50 years as an alternative treatment of uncomplicated urinary tract infections (UTIs). ${ }^{3}$ The same result is now being feared with the increased use of NFT in recent years.

The major strengths of NFT are its action at multiple sites and levels, its high urinary concentration, safety in pregnancy, being used orally, and well tolerability with side-effects occurring at rates $<0.001 \% .4$. 4 At high concentrations, NFT is converted by bacterial nitroreductases to highly reactive electrophilic intermediate that binds nonspecifically to ribosomal proteins and rRNA and causes complete cessation of synthesis of bacterial DNA, RNA, and proteins. It also inhibits bacterial enzymes such as $\beta$-galactosidase at concentrations near MICs and disrupts bacterial metabolism in absence of reductive activation of this drug. ${ }^{6}$

NFT has a broad-spectrum activity against the main uropathogens (Escherichia coli [E. coli], Citrobacter spp., group B Streptococci, Enterococci, Staphylococcus aureus, Staphylococcus epidermidis, Klebsiella pneumonia [K. pneumoniae], and Enterobacter spp.) and has shown to be active against extended spectrum $\beta$-lactamase (ESBL)-producing Enterobacteriaceae and vancomycin-resistant enterococci. ${ }^{3}$ However, Proteus, Providencia, Morganella, Serratia, Acinetobacter, and Pseudomonas are highly resistant to this drug. ${ }^{4}$

Detection of UTI causing pathogens and analyzing resistance pattern of these pathogens to antimicrobials is crucial and valuable in improving the efficacy of empirical treatment and preventing emergence of high resistance. ${ }^{7}$ This will also have an impact on morbidity, mortality, and cost of treatment especially in developing countries like India. It is important to understand that the resistance pattern observed may vary from hospital to community, large hospital to small hospital, state to state, and country to country. ${ }^{8}$ Since most of the UTIs are treated empirically, the antimicrobial agent prescribed should be determined by expected susceptibility pattern. Hence our study was undertaken to determine the susceptibility pattern of NFT in gram-negative urinary isolates in a tertiary care hospital in North India and to evaluate their bacteriological and epidemiological profile along with co-existing resistance to other important urinary antimicrobials.

\section{Material and Methods}

\section{Setting}

This retrospective study was conducted in a tertiary care hospital in North India.

\section{Subjects}

The highest nitrofurantoin resistance (NFT-R) reported from India was found to be around $48 \%{ }^{9}$ Taking the mentioned reference as the prevalence for NFT-R, a sample size of 500 gram-negative bacterial urinary isolates was taken.

\section{Methodology}

Records of antimicrobial susceptibility were reviewed from July to September 2019 for gram-negative urinary isolates obtained from patients suspected of UTI. Antimicrobial susceptibility was performed using the Kirby-Bauer disk diffusion method on Mueller Hinton agar and interpreted using CLSI 2019. Test for ESBL producers was done using double disk approximation test. Informed consent was taken from the patients for usage of data in the study. Only one isolate per patient was included. Only growths with significant colony count ( $>10^{5} \mathrm{cfu} / \mathrm{mL}$ ) were included for the data analysis.

The susceptibility results for the following antibiotics were evaluated: ampicillin $(10 \mu g)$, norfloxacin $(5 \mu \mathrm{g})$, ciprofloxacin $(5 \mu \mathrm{g})$, NFT (300 $\mu \mathrm{g})$, gentamicin $(10 \mu \mathrm{g})$, ceftazidime $(30 \mu \mathrm{g})$, piperacillin-tazobactam $(100 / 10 \mu \mathrm{g})$, meropenem $(10 \mu \mathrm{g})$, ertapenem $(10 \mu \mathrm{g})$, and imipenem $(10 \mu \mathrm{g})$.

\section{Analysis}

Data analysis was performed using the SPSS windows version 25.0 software. Test of significance like chi-square test was applied to find out the results. A value of $p<0.05$ was considered to be statistically significant. Data obtained from this study was also analyzed using descriptive statistics such as percentage and proportion.

\section{Results}

Among the total 500 isolates, majority (64\%) were E. coli, followed by Klebsiella sp. (26\%), Pseudomonas aeruginosa (4.6\%), Acinetobacter sp. (2\%), and Proteus sp. (1.8\%). Among the minority were Citrobacter sp., Morganella morganii, and Enterobacter sp. (-Table 1). Pseudomonas aeruginosa, Morganella morganii, and Proteus sp. are considered to be intrinsically resistant and constituted $6.74 \%$ (34) of the total isolates.

\section{Nitrofurantoin Resistance Profile}

NFT-R was seen in $20.17 \%$ (94) of the total isolates excluding the intrinsic resistant organisms, whereas 9.01\% (42) were intermediate (I). Highest resistance was seen in Klebsiella sp. (44.61\%) and E. coli (8.12\%). High resistance was also seen in Acinetobacter sp. (80\%). Five Citrobacter sp. were isolated; two of them were found to be NFT-R and one was intermediate sensitive. Only one Enterobacter sp. was isolated which was found to be NFT sensitive (-Table 2 ). 
Table 1 Distribution of gram-negative isolates $(N=500)$

\begin{tabular}{|l|l|l|l|}
\hline S. no. & Organism & No. & $\%$ \\
\hline 1 & E. coli & 320 & 64.00 \\
\hline 2 & Klebsiella sp. & 130 & 26.00 \\
\hline 3 & Pseudomonas sp. & 23 & 4.60 \\
\hline 4 & Acinetobacter sp. & 10 & 2.00 \\
\hline 5 & Proteus mirabilis & 7 & 1.40 \\
\hline 6 & Citrobacter sp. & 5 & 1.00 \\
\hline 7 & Morganella morganii & 2 & 0.40 \\
\hline 8 & Proteus vulgaris & 2 & 0.40 \\
\hline 9 & Enterobacter sp. & 1 & 0.20 \\
\hline Total & & 500 & 100.00 \\
\hline
\end{tabular}

Table 2 Nitrofurantoin resistance profile

\begin{tabular}{|l|l|l|l|l|l|}
\hline $\begin{array}{l}\text { Nitrofurantoin } \\
(\%)\end{array}$ & $\begin{array}{l}\text { Total } \\
(\boldsymbol{n}=\mathbf{4 6 6 )}\end{array}$ & $\begin{array}{l}\text { E. coli } \\
(\boldsymbol{n}=\mathbf{3 2 0})\end{array}$ & $\begin{array}{l}\text { Klebsiella sp. } \\
(\boldsymbol{n}=130)\end{array}$ & $\begin{array}{l}\text { Acinetobacter sp. } \\
(\boldsymbol{n}=10)\end{array}$ & $\begin{array}{l}\text { Citrobacter sp. } \\
(\boldsymbol{n}=5)\end{array}$ \\
\hline $\begin{array}{l}\text { Intermediate } \\
\text { sensitive }\end{array}$ & $9.01(42)$ & $0.31(20)$ & $15.38(20)$ & $10(1)$ & $20(1)$ \\
\hline Resistant & $20.17(94)$ & $8.12(26)$ & $44.61(58)$ & $80(8)$ & $40(2)$ \\
\hline$p$-Value & & 0.02 & 0.12 & 0.17 & 0.67 \\
\hline
\end{tabular}

Of the isolates that were resistant or intermediate, $71.18 \%$ were adults (> 12 years of age) and $28.82 \%$ were pediatric. Majority were females (64.69\%). Resistance was seen highest in patients admitted in wards (57.74\%), whereas $39.29 \%$ isolates were from patients of various OPDs, and only $2.95 \%$ isolates were from ICU patients ( - Table $\mathbf{3}$ ).

\section{Co-existing Resistance}

Among NFT-R E. coli, high resistance was also seen against ampicillin (92.30\%), cefazolin (88.46\%), ceftazidime (73.0\%), and fluroquinolones (65.38\%). Among the carbapenems, imipenem (57.69\%) showed the highest resistance, followed by ertapenem (42.30\%), and least resistant was meropenem (19.23\%). ESBL production was seen in $30.76 \%$ isolates. Less resistant drugs were piperacillin-tazobactam (26.92\%) and gentamicin (30.76\%).

Similarly, among NFT-R Klebsiella sp., high resistance was seen against ampicillin (94.18\%), cefazolin (74.13\%), ceftazidime (62.06\%), and fluroquinolones (53.44\%). Among the carbapenems, ertapenem showed highest resistance (50\%), followed by imipenem (46.55\%) and meropenem (39.65\%). ESBL production was seen in $12.06 \%$ isolates. Less resistant drugs were piperacillin-tazobactam (46.55\%) and gentamicin (46.55\%) (-Table 4).

Carbapenemase resistance among NIT-R E. coli and Klebsiella sp. was 57.15\% (48/84).

\section{Discussion}

The rapid development and spread of antimicrobial resistance among gram-negative bacteria have become a major public health concern. This study highlights an update on the susceptibility profile of NFT in gram-negative uropathogens and co-existing resistance to other commonly used antimicrobials.

Recently, there has been a new interest for older antibiotics due to alterations in pathogen distribution and resistance. NFT is a synthetic nitrofuran antimicrobial agent that has been used for years and still considered to be active against most of the uropathogens including the multiresistant strains. ${ }^{5}$ In many studies, NFT has been the drug with least resistance against E. coli. ${ }^{10,11}$ In the year 2011, IDSA recommended NFT as the drug of choice for empirical treatment of uncomplicated UTIs. ${ }^{12}$ Since then there has been an increase in consumption of NFT which might result in increased selection pressure for resistant strains and overall increase in resistance.

The study demonstrates that $E$. coli remains the leading uropathogen being responsible for $64 \%$ of UTIs in our area. This is consistent with findings of other studies in which $E$. coli was the most frequently reported isolate from patients with community-acquired UTIs. ${ }^{9-11,13}$

In our study, the overall rate of resistance of NFT was $20.17 \%$ among various gram-negative uropathogens and $9.01 \%$ was intermediate sensitive according to CLSI 2019 guidelines. NFT-R among Klebsiella sp. was highest $(44.61 \%)$ whereas $8.12 \%$ of $E$. coli isolates were resistant and the result was found to be statistically significant. NFT-R pattern has been seen to vary greatly among different geographical areas especially in a vast country like India. In a study conducted by Shaifali et al in 2012, NFT-R was reported to be $13 \%$ in E. coli and $7 \%$ in Klebsiella sp. ${ }^{10}$ In another study conducted by Sood and Gupta, NFT-R among E. coli was 5 to $6 \%$ whereas $61.2 \%$ resistance was reported in other gram-negative enteric bacteria. Highest resistance (94.44\%) was reported for nonfermenting gram-negative 
Table 3 Epidemiological distribution of NFT-R and NFT-I isolates

\begin{tabular}{|l|l|l|l|}
\hline \multirow{2}{*}{ Age } & Adults ( $\geq 12$ y of age) & \multicolumn{1}{|c|}{ Pediatrics (< 12 y of age) } \\
\cline { 2 - 4 } & $71.18 \%$ & \multicolumn{2}{|c|}{$28.82 \%$} \\
\hline \multirow{2}{*}{ Sex } & Male & & \multicolumn{2}{|c|}{ Female } \\
\cline { 2 - 4 } & $35.31 \%$ & OPDs & $64.69 \%$ \\
\hline \multirow{2}{*}{ Location } & Inpatient (excluding ICUs) & $39.29 \%$ & $2.95 \%$ \\
\hline
\end{tabular}

Abbreviations: ICU, intensive care unit; NFT-I, nitrofurantoin intermediate; NFT-R, nitrofurantoin resistance; OPD, outpatient departments.

Table 4 Co-existing resistance (\% resistance)

\begin{tabular}{|l|l|l|l|l|l|l|l|l|l|l|}
\hline NIT-R isolates & Amp & CZ & CAZ & FQs & PIT & Genta & MRM & IPM & ERT & ESBL \\
\hline E. coli $(n=26)$ & 92.30 & 88.46 & 73.07 & 65.38 & 26.92 & 30.76 & 19.23 & 57.69 & 42.30 & 30.76 \\
\hline $\begin{array}{l}\text { Klebsiella } \\
(n=58)\end{array}$ & 94.82 & 74.13 & 62.06 & 53.44 & 46.55 & 46.55 & 39.65 & 46.55 & 50 & 12.06 \\
\hline
\end{tabular}

Abbreviations: Amp, ampicillin; ESBL, extended spectrum $\beta$-lactamase; NFT, nitrofurantoin.

bacteria. ${ }^{11}$ The wide variation in resistance rate might be due to different local prescribing practices, with high resistance seen with high prescription and difference in existing resistance pattern in the areas. Various NFT-R patterns reported all around India are listed in - Table $\mathbf{5}$.

Interestingly, in Western countries, resistance is still rare in E. coli and most other ESBL-producing Enterobacteriaceae. A population-based survey of in vitro antimicrobial resistance of urinary E. coli isolates among U.S. outpatients from 2000 to 2010 showed NFT-R from 0.8 to $1.6 \%{ }^{14}$ The susceptibility data from E. coli community-acquired UTIs in Europe points to a similar prevalence of low resistance ( $2 \%$ from isolates in 2007-2008). ${ }^{15}$

Even in earlier eras of widespread use, baseline NFT-R was low $(0-5 \%)$, likely because of multiple modes of action and the acquisition or emergence of resistance being relatively uncommon (approximately 10-7/cell for E. coli). ${ }^{16,17}$ NFT-R is thought to be due to loss of intracellular nitroreductase activity via stepwise mutations in the DNA regions encoding the enzymes (nsfA and $n s f B$ ) and the deletion in ribE (encoding lumazine synthase involved in biosynthesis of flavin mononucleotide). ${ }^{16}$ In 2003, a plasmid-encoded efflux pump mutation, OqxAB, was also detected to be an important NFT-R determinant. ${ }^{18}$

High resistance was also seen in Acinetobacter sp. (80\%), but many studies have reported Acinetobacter sp., Pseudomonas aeruginosa, Morganella morganii, Proteus sp., and Providencia to be intrinsically resistant.,4

Majority of resistant isolates belonged to females (64.69\%), which is consistent with the fact that UTI occurs more commonly in females than males due to structural differences. Approximately $80 \%$ of all UTIs occur in women. ${ }^{11}$ Resistance was seen highest in patients admitted in wards (57.74\%) whereas $39.29 \%$ isolates were from patients of various OPDs and only $2.95 \%$ isolates were from ICUs patients. High level NFT-R is found to be associated with surgical wards and ICUs correlated with the use of invasive urinary catheters/procedures. ${ }^{19}$ Any age correlation with resistance pattern has not been observed.

Similar results were found in terms of co-existing resistance. Gram-negative bacteria are now highly resistant to oral drugs such as aminopenicillins, ciprofloxacin, norfloxacin, and cotrimoxazole. ${ }^{10,11,13}$ ESBL production was seen in $30.76 \%$ of $E$. coli and $12.06 \%$ of Klebsiella NFT-R isolates. Other study also revealed overall $23.83 \%$ of $E$. coli isolates and $8.69 \%$ of Klebsiella isolates to be ESBL producers. ${ }^{11}$ Alternatively, one study evaluated NIT against multidrug-resistant Enterobacteriaceae and found NIT to be $70 \%$ effective against ESBLs and a low sensitivity rate for metallo- $\beta$-lactamases (38\%) and AmpC $\beta$-lactamases (32\%). ${ }^{3}$ NIT susceptibility profile has also been evaluated for carbapenem-resistant Enterobacteriaceae (CRE) and found to be only 56\% effective for CR E. coli. ${ }^{1}$

Table 5 Various NFT resistance patterns reported all around India

\begin{tabular}{|l|l|l|l|l|}
\hline Study & Year & Place & \multicolumn{2}{|c|}{ Resistance $\%$} \\
\cline { 4 - 5 } & & & Escherichia coli & Klebsiella sp. \\
\hline Kothari and Sagar $^{21}$ & 2005 & Delhi & $24.4 \%$ & NE \\
\hline Sood and Gupta & & Jaipur, Rajasthan & $5-6 \%$ & NE \\
\hline Shaifali et al & $2007-2009$ & Lucknow, Uttar Pradesh & $13 \%$ & $9 \%$ \\
\hline Badhan et al $^{13}$ & 2011 & Punjab & $6 \%$ & $21 \%$ \\
\hline Kulkarni et al $^{20}$ & $2012-2014$ & Karnataka & $<8 \%$ & NE \\
\hline Suresh et al & $2012-2015$ & Ooty, South India & $8.3 \%$ & $<1 \%$ \\
\hline Patel et al & $2015-2016$ & Gujarat & $27.7 \%$ & $48.3 \%$ \\
\hline
\end{tabular}

Abbreviations: NE, not evaluated; NFT, nitrofurantoin. 
In our study, coexisting rate of CRE was found to be $57.7 \%$ for NIT-R E. coli and $56.9 \%$ for NIT-R Klebsiella sp. One study reported a low-level resistance (3.29\%) for E. coli isolates. ${ }^{20}$ Study conducted by Patel et al reported overall rates of CRE to be $8.1 \%$ and $24.3 \%$ for E. coli and Klebsiella sp., respectively. ${ }^{9}$

The major limitation of this study is that NIT MICs could not be performed, and it does not take into account risk factors that can cause drug resistant and complicated UTIs such as diabetes, compromised immunity, cancer chemotherapy, HIV, prolonged urinary catheterization, recent antibiotic use, or incomplete treatment of prior UTIs.

\section{Conclusion}

NFT appears to have better efficacy than aminopenicillins, ciprofloxacin, and norfloxacin. An agent is deemed unacceptable for empiric treatment where the rate of resistance exceeds $20 \% .^{12}$ This highlights the need to maintain strict vigilance and regular monitoring of NIT resistance pattern. Taking into consideration the importance of NFT in acute uncomplicated UTIs and its efficacy to manage MDR infections, increased care should be taken in the prescription of NFT to avoid further increase of NFT-R among Enterobacteriaceae. Also, species identification and antibiotic susceptibility testing of pathogens are necessary to avoid prescribing NFT for organisms intrinsically resistant to NFT. The stewardship of NFT is necessary to prolong its usefulness for uncomplicated UTIs.

\section{Funding \\ None.}

\section{Conflict of Interest}

None declared.

\section{Acknowledgment}

No financial support was received and recognition of personal assistance for the work being published is mentioned in the title page. No sources of outside support exist for the paper.

\section{References}

1 Amladi AU, Abirami B, Devi SM, et al. Susceptibility profile, resistance mechanisms \& efficacy ratios of fosfomycin, nitrofurantoin \& colistin for carbapenem-resistant Enterobacteriaceae causing urinary tract infections. Indian J Med Res 2019;149(2):185-191

2 Kahlmeter G; ECO.SENS. An international survey of the antimicrobial susceptibility of pathogens from uncomplicated urinary tract infections: the ECO.SENS Project. J Antimicrob Chemother 2003;51(1):69-76

3 Singh RM, Devi MU, Singh KL, Singh HL, Keisham C, Singh KH. Evaluation of nitrofurantoin activity against the urinary isolates in the current scenario of antimicrobial resistance. Ann Trop Med Public Health 2015;8(6):280-285

4 Shakti L, Veeraraghavan B. Advantage and limitations of nitrofurantoin in multi-drug resistant Indian scenario. Indian J Med Microbiol 2015;33(4):477-481
5 Munoz-Davila MJ. Role of old antibiotics in the era of antibiotic resistance. Highlighted nitrofurantoin for the treatment of lower urinary tract infections. Antibiotics (Basel) 2014;3(1):39-48

6 Jorgensen J. Manual of Clinical Microbiology. 11th ed. Washington, DC: American Society of Microbiology;2015

7 Gupta V, Yadav A, Joshi RM. Antibiotic resistance pattern in uropathogens. Indian J Med Microbiol 2002;20(2):96-98

8 Farajnia S, Alikhani MY, Ghotaslou R, Naghili B, Nakhlband A. Causative agents and antimicrobial susceptibilities of urinary tract infections in the northwest of Iran. Int J Infect Dis 2009;13(2):140-144

9 Patel HB, Soni ST, Bhagyalaxmi A, Patel NM. Causative agents of urinary tract infections and their antimicrobial susceptibility patterns at a referral center in Western India: an audit to help clinicians prevent antibiotic misuse. J Family Med Prim Care 2019;8(1):154-159

10 Shaifali I, Gupta U, Mahmood SE, Ahmed J. Antibiotic susceptibility patterns of urinary pathogens in female outpatients. N Am J Med Sci 2012;4(4):163-169

11 Sood S, Gupta R. Antibiotic resistance pattern of community acquired uropathogens at a tertiary care hospital in Jaipur, Rajasthan. Indian J Community Med 2012;37(1):39-44

12 Gupta K, Hooton TM, Naber KG, et al. Infectious Diseases Society of America; European Society for Microbiology and Infectious Diseases. International clinical practice guidelines for the treatment of acute uncomplicated cystitis and pyelonephritis in women: a 2010 update by the Infectious Diseases Society of America and the European Society for Microbiology and Infectious Diseases. Clin Infect Dis 2011;52(5):e103-e120

13 Badhan R, Singh DV, Badhan LR, Kaur A. Evaluation of bacteriological profile and antibiotic sensitivity patterns in children with urinary tract infection: a prospective study from a tertiary care center. Indian J Urol 2016;32(1):50-56

14 Sanchez GV, Master RN, Karlowsky JA, Bordon JM. In vitro antimicrobial resistance of urinary Escherichia coli isolates among U.S. outpatients from 2000 to 2010. Antimicrob Agents Chemother 2012;56(4):2181-2183

15 Kahlmeter G, Poulsen HO. Antimicrobial susceptibility of Escherichia coli from community-acquired urinary tract infections in Europe: the ECO.SENS study revisited. Int J Antimicrob Agents 2012;39(1):45-51

16 Huttner A, Verhaegh EM, Harbarth S, Muller AE, Theuretzbacher U, Mouton JW. Nitrofurantoin revisited: a systematic review and meta-analysis of controlled trials. J Antimicrob Chemother 2015;70(9):2456-2464

17 Sandegren L, Lindqvist A, Kahlmeter G, Andersson DI. Nitrofurantoin resistance mechanism and fitness cost in Escherichia coli. J Antimicrob Chemother 2008;62(3):495-503

18 Ho P-L, Ng K-Y, Lo W-U, et al. Plasmid-mediated OqXAB is an important mechanism for nitrofurantoin resistance in Escherichia coli. Antimicrob Agents Chemother 2015;60(1):537-543

19 Osei Sekyere J. Genomic insights into nitrofurantoin resistance mechanisms and epidemiology in clinical Enterobacteriaceae. Future Sci OA 2018;4(5):FSO293

20 Kulkarni SR, Peerapur BV, Sailesh KS. Isolation and antibiotic susceptibility pattern of Escherichia coli from urinary tract infections in a Tertiary Care Hospital of North Eastern Karnataka. J Nat Sci Biol Med 2017;8(2):176-180

21 Kothari A, Sagar V. Antibiotic resistance in pathogens causing community-acquired urinary tract infections in India: a multicenter study. J Infect Dev Ctries 2008;2(5):354-358

22 Suresh A. Antibiotic resistance pattern in urinary tract infection during pregnancy in South Indian population. Asian J Pharm 2018;12(2) 